\title{
Variabilidad de las indicaciones en el diagnóstico prenatal del síndrome de Down
}

\author{
Andrés Fandiño-Losada ${ }^{1,2}$, Beatriz E. Lucumí-Villegas 1,3, Julián Ramírez-Cheyne 1,4, \\ Carolina Izasa de Lourido 1,4,5, Wilmar Saldarriaga. 1,4,6,7 \\ ${ }^{1}$ Universidad del Valle, Cali, Colombia. ${ }^{2}$ Profesor Escuela de Salud Pública, Facultad de Salud, Universidad del Valle. \\ ${ }^{3}$ Alumna de Medicina, Universidad del Valle. ${ }^{4}$ Profesor Departamento de Morfología, Escuela de Ciencias Básicas, \\ Facultad de Salud, Universidad del Valle. 5 Laboratorio de Genética Médica “Carolina Isaza”, Clínica DIME. ${ }^{2}$ Departamento \\ de Ginecología y Obstetricia, Escuela de Medicina, Universidad del Valle. ${ }^{7}$ Gineco-Obstetra, Hospital Universitario del \\ Valle "Evaristo García". Cali, Colombia.
}

\section{RESUMEN}

Introducción: Para lograr el diagnostico prenatal de síndrome de Down (SD) los protocolos han evolucionado a través de la construcción de indicaciones para realizar procedimientos invasivos que con pruebas citogenéticas o moleculares confirman el diagnostico in útero. Objetivo: El objetivo de este estudio es analizar y caracterizar las indicaciones de los procedimientos invasivos prenatales a los que se les realizó cariotipo y específicamente a las que tuvieron diagnóstico de síndrome de Down. Método: Estudio de diseño transversal para caracterizar las indicaciones de procedimientos invasivos en diagnóstico prenatal en un centro de diagnóstico genético en Cali, Colombia. Resultados: Se analizaron 623 casos (enero de 2013 - febrero de 2015). Del total de procedimientos invasivos prenatales analizados, el $7,1 \%$ fueron diagnosticados SD. La indicación con mayor frecuencia fueron los hallazgos de malformaciones en los exámenes ecográficos $(45,4 \%)$; la indicación con mayor valor predictivo positivo fue la sonolucencia más otro marcador ecográfico $(60,0 \%)$. Además, se encontró un mayor valor predictivo positivo de los procedimientos invasivos para diagnosticar SD en el grupo de edad entre 35 a 39 años $(21,6 \%)$ en comparación con el grupo de 40 años y mayores $(4,9 \%)$; esto probablemente secundario a que las indicaciones del procedimiento invasivo en el primer grupo eran diferentes a edad materna. Conclusiones: En Colombia, la primera ayuda diagnóstica para sospechar el SD in útero es la ecografía de segundo trimestre. La edad materna, la ansiedad y la sonolucencia nucal alterada también juegan un rol importante en este tipo de diagnóstico.

\section{PALABRAS CLAVE: Síndrome de Down, diagnóstico prenatal, procedimientos invasivos, cariotipo}

\section{SUMMARY}

Introduction: For prenatal diagnosis of Down syndrome (DS), the protocols have evolved through the construction of indications for invasive procedures through karyotyping or molecular tests confirmed the diagnosis in utero. Objective: To analyze and characterize the indications of prenatal invasive procedures that are performed karyotype and specifically those who had diagnosis of (DS) in Cali, Colombia. Methods: Crossover design study to characterize signs of invasive diagnostic procedures in prenatal genetic diagnosis center in Cali. 623 cases were analyzed (January 2013 - February 2015). Results: Of all performed invasive procedures, $7.1 \%$ were diagnosed with DS. The most common indications were the findings of malformations in ultrasound examinations ( $45.4 \%)$; the indication with the highest positive predictive value was the nuchal translucency with other sonographic marker (60.0\%). In addition, a higher positive predictive value of invasive diagnostic procedures for SD was found, in the age group between 35-39 years (21.6\%) 
compared with the group of 40 and older (4.9\%); this probably secondary to the invasive procedure directed in the first group were different maternal age. Conclusions: In Colombia, first help to suspect diagnosed DS in utero is second trimester ultrasound. Maternal age, anxiety and impaired nuchal translucency also play an important role in this diagnosis.

\section{KEY WORDS: Down syndrome, prenatal diagnosis, invasive procedures, karyotype}

\section{INTRODUCCIÓN}

El síndrome de Down (SD) es causado por la presencia de un fragmento extra del cromosoma 21, 21q22.12 a 22.2 (1). El 95\% de los casos se presentan por un cromosoma 21 completo adicional, produciendo una trisomía 21 libre. El resto de los pacientes tienen extra solo la región crítica del cromosoma 21 (2).

Es la alteración cromosómica más frecuente en nacidos vivos, ocurre en 1 de cada 600 a 800 nacimientos (3). Los pacientes afectados tienen un fenotipo que incluye retardo mental, baja talla, obesidad, facie característica, defectos cardiovasculares, deficiencias inmunológicas, pliegue palmar único, hipotonía, hipotiroidismo, malformaciones esqueléticas, entre otros (4). En la actualidad, se han considerado 3 factores de riesgo como probables agentes etiológicos de la trisomía 21, como son las anomalías cromosómicas de los padres capaces de inducir una no disyunción meiótica secundaria, la exposición precigótica materna a radiaciones ionizantes y la edad materna avanzada (5).

El diagnóstico prenatal confirmatorio del SD se realiza a través del cariotipo (FISH, MLPA o Hibridación genómica comparativa), analizando muestras obtenidas de alguno de los productos de la gestación a través de biopsia de vellosidad corial, amniocentesis o cordocentesis $(6,7)$. Las estrategias de detección convencionales suelen clasificar a las pacientes como de alto y bajo riesgo para que su gestación tenga SD, esta clasificación suele sustentarse en edad materna, pruebas no invasivas realizadas en sangre materna y por marcadores ecográficos de alteraciones cromosómicas. Recientemente se ha implementado pruebas moleculares que analizan DNA fetal en sangre materna y que diagnostican SD con sensibilidad y especificidad superiores al $99 \%(7,8)$. Según los resultados de estas pruebas y en consenso con los padres se toma la decisión de realizar o no un procedimiento invasivo teniendo en cuenta el riesgo de pérdida del embarazo secundario al procedimiento.
El objetivo de este estudio es analizar y caracterizar las indicaciones de los procedimientos invasivos prenatales que se les realizo cariotipo y específicamente a las que tuvieron diagnóstico de $\mathrm{SD}$, en un laboratorio de referencia de citogenética de la ciudad de Cali, Colombia.

\section{MATERIALES Y MÉTODOS}

Estudio de diseño transversal para caracterizar las indicaciones de amniocentesis, biopsia de vellosidad coriónica o cordocentesis en un centro de diagnóstico genético en Cali, Colombia. Los sujetos de estudio corresponden a todas las muestras de embarazos, que se procesaron en un instituto de genética médica de Cali entre el 1 de enero de 2013 y el 28 de febrero de 2015. En ese período se procesaron 738 muestras, de las cuales se descartaron $115(15,6 \%)$, por falta de datos sobre la indicación del examen o la edad materna, de estos ninguno tuvo diagnóstico de SD. El diagnóstico invasivo se realizó mediante cordocentesis, amniocentesis, biopsia de vellosidad coriónica y el cariotipo se realizó con tinción de Giemsa obteniéndose un mínimo de resolución de 450 bandas.

Para realizar el análisis, se dividieron las edades de las embarazadas según tres grupos de edad 1) igual o menor de 34 años 2) entre 35 a 39 años y 3) 40 o más años, de acuerdo al riesgo de cromosopatía según la literatura (5). Además, la indicación médica para la realización de cariotipo en el embarazo, se agruparon en las categorías que aparecen en la Tabla I.

El análisis estadístico utilizó dos abordajes: 1) En el grupo de pacientes con examen final positivo para Trisomía 21, se determinó la dependencia entre el tipo de indicación para examen de cromosomopatía y los grupos de edad mediante la prueba exacta de Fisher (Tabla I), y excluyendo el grupo de edad mayor y la indicación por edad. 2) Se calculó el Valor Predictivo Positivo y el Intervalo de Confianza al 95\% (IC95\%) de cada indicación de examen invasivo para el diagnóstico de Trisomía 21. 


\section{Tabla I \\ INDICACIONES DE PROCEDIMIENTOS INVASIVOS PARA EL DIAGNÓSTICO DE SÍNDROME DE DOWN}

\begin{tabular}{|c|c|c|c|c|c|c|c|c|c|}
\hline \multirow[t]{2}{*}{ Indicación de diagnóstico invasivo } & \multicolumn{2}{|c|}{$\leq 34$ años } & \multicolumn{2}{|c|}{ 35-39 años } & \multicolumn{2}{|c|}{$\geq 40$ años } & \multicolumn{2}{|c|}{ Total } & \multirow{2}{*}{$\begin{array}{l}\text { Pruebas } \\
\text { realizadas* }\end{array}$} \\
\hline & $\mathrm{n}$ & $\%$ & $\mathrm{n}$ & $\%$ & $\mathrm{n}$ & $\%$ & $\mathrm{n}$ & $\%$ & \\
\hline Malformación en ECO** & 8 & 57,1 & 9 & 42,9 & 3 & 33,3 & 20 & 45,4 & 306 \\
\hline $\mathrm{SN}^{\star \star \star}+$ otro marcador $\mathrm{ECO}$ & 2 & 14,3 & 2 & 9,5 & 0 & 0,0 & 4 & 9,1 & 7 \\
\hline Solo SN alterada & 2 & 14,3 & 3 & 14,3 & 0 & 0,0 & 5 & 11,4 & 75 \\
\hline Edad materna & 0 & 0,0 & 0 & 0,0 & 6 & 66,7 & 6 & 13,6 & 140 \\
\hline Ansiedad & 1 & 7,1 & 4 & 19,1 & 0 & 0,0 & 5 & 11,4 & 43 \\
\hline Antecedente de alteración cromosómica & 0 & 0,0 & 1 & 4,8 & 0 & 0,0 & 1 & 2,3 & 23 \\
\hline 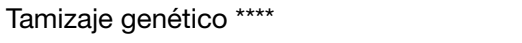 & 1 & 7,1 & 2 & 9,5 & 0 & 0,0 & 3 & 6,8 & 23 \\
\hline Tres o más abortos previos & 0 & 0,0 & 0 & 0,0 & 0 & 0,0 & 0 & 0,0 & 6 \\
\hline Total & 14 & 100 & 21 & 100 & 9 & 100 & 44 & 100 & 623 \\
\hline
\end{tabular}

* Número de pruebas realizadas, incluyendo pruebas negativas. ${ }^{\star \star E C O: ~ E c o g r a f i ́ a . ~}{ }^{\star \star \star}$ SN: Sonolucencia nucal. ${ }^{\star \star * \star *}$ Tamizaje genético en primer trimestre incluye: ecografia semana 11 a 14 de gestación, cuantificación de la fracción libre de la Beta HCG, proteína plasmática asociada al embarazo. Prueba de Fisher de Independencia para toda la tabla: 0,005. Prueba de Fisher de Independencia, sin incluir la indicación por edad materna: 0,975.

\section{RESULTADOS}

En este estudio, se accedió a la información sobre 623 mujeres gestantes con alguna indicación para el diagnóstico prenatal invasivo de alteraciones cromosómicas, de las cuales $9(1,2 \%)$ fueron embarazos gemelares, pero a ninguno de aquellos se les tomó dos muestras. Estos procedimientos invasivos se dividieron de la siguiente manera según el tipo de procedimiento invasivo: amniocentesis: 547 , biopsia de vellosidad coriónica: 53, cordocentesis: 23. Además, se hicieron analisis según edad de la madre y estas muestras se dividieron en gestantes de 34 años o menos 331 (53,1\%), gestantes de 35 a 39 años de edad: 110 (17,7\%), gestantes de 40 años o más: $182(29,2 \%)$. Del total de los exámenes invasivos realizados, 44 (7,1\%; IC95\% 5,2\%-9,4\%) tuvieron un diagnóstico de SD en el cariotipo.

El procedimiento invasivo prenatal con el que se diagnosticó SD se realizó en el primer trimestre en 5 casos $(11,4 \%)$, en segundo trimestre en 37 casos $(84,1 \%)$ y en tercer trimestre en 2 casos $(4,5 \%)$.

En la Tabla II, se presenta la distribución porcentual de las indicaciones de los procedimientos invasivos prenatales para el diagnóstico de alteraciones cromosómicas incluyendo SD. El análisis de esa tabla indica que existe una asociación entre la indicación para el diagnóstico final de Trisomía 21 y el grupo de edad de madres de 40 años y más, a expensas de la indicación por la edad materna (Prueba de Fisher, $p=0,005$ ).
Como este resultado es tautológico, entonces se excluyó ese grupo de edad y se repitió al análisis de dependencia, con un resultado de la prueba exacta de Fisher de $p=0,975$, mostrando que no existe una asociación entre los dos grupos de gestantes menores a 40 años de edad y la indicación de cariotipo por procedimiento invasivo en los fetos que tuvieron un resultado positivo de Trisomía 21.

\section{DISCUSIÓN}

El diagnóstico prenatal de SD y de otras anomalías cromosómicas se ha realizado por más de 40 años y durante todo el tiempo ha evolucionado mejorando los criterios para disminuir el número de procedimientos invasivos necesarios para lograr el diagnóstico, todo esto de la mano del avance de la ecografía prenatal y de las técnicas moleculares para la cuantificación de proteínas y análisis de ADN. Durante los años 70's, la edad materna sobre los 35 años era el único factor de riesgo usado para sospechar anomalías cromosómicas y sugerir a las pacientes un procedimiento invasivo como la amniocentesis para realizar un cariotipo en líquido amniótico y hacer el diagnóstico. Con esta metodología se lograba diagnosticar menos de un tercio de los casos de SD; además, de todas esas mujeres mayores de 35 años sometidas al procedimiento, solo el $2 \%$ tendría una alteración cromosómica en el cariotipo (9). 


\section{Tabla II \\ VALOR PREDICTIVO POSITIVO PARA EL DIAGNÓSTICO DE SÍNDROME DE DOWN DE LAS INDICACIONES DE PROCEDIMIENTO INVASIVO}

\begin{tabular}{|c|c|c|c|c|c|}
\hline \multirow{2}{*}{$\begin{array}{l}\text { Indicación de diagnóstico } \\
\text { invasivo }\end{array}$} & $\leq 30$ años & 35 a 39 años & $\geq 40$ años & Total & Fisher \\
\hline & $\%(I C 95 \%)$ & $\%(I C 95 \%)$ & $\%(I C 95 \%)$ & $\%(I C 95 \%)$ & $p=$ \\
\hline Malformación en ECO* & $3,5(1,5-6,7)$ & $19,2(9,1-33,3)$ & $10,7(2,3-28,2)$ & $6,5(4,0-9,9)$ & 0,001 \\
\hline $\begin{array}{l}\mathrm{SN}^{\star \star}+\text { otro marcador } \\
\text { en } \mathrm{ECO} \text { de primer } \\
\text { trimestre }\end{array}$ & $50,0(6,8-93,2)$ & $100,0(15,81-100)$ & $0,0(0-97,5)$ & $60,0(14,7-94,7)$ & 0,429 \\
\hline Solo SN alterada en ECO & $3,9(0,5-13,2)$ & $16,7(3,6-41,4)$ & $0,0(0-52,2)$ & $6,7(2,2-14,9)$ & 0,177 \\
\hline Edad materna & NA & NA & $4,3(1,6-9,1)$ & $4,3(1,6-9,1)$ & NA \\
\hline Ansiedad & $2,6(0,1-13,5)$ & $40,0(12,2-73,8)$ & NA & $10,2(3,4-22,2)$ & 0,004 \\
\hline $\begin{array}{l}\text { Antecedente de } \\
\text { alteración cromosómica }\end{array}$ & $0,0(0,0-28,5)$ & $16,7(0,4-64,1)$ & $0,0(0-45,9)$ & $4,4(0,1-22,0)$ & 0,522 \\
\hline Tamizaje genético*** & $10,0(0,3-44,5)$ & $22,2(2,8-60,0)$ & $0,0(0-60,2)$ & $13,0(2,8-33,6)$ & 0,771 \\
\hline $\begin{array}{l}\text { Tres o más abortos } \\
\text { previos }\end{array}$ & $0,0(0-97,5)$ & $0,0(0-60,2)$ & $0,0(0-97,5)$ & $0,0(0-45,9)$ & NA \\
\hline$\overline{\text { Total }}$ & $4,1(2,2-6,8)$ & $22,1(14,2-31,8)$ & $4,9(2,2-9,0)$ & $7,1(5,2-9,4)$ & 0,000 \\
\hline
\end{tabular}

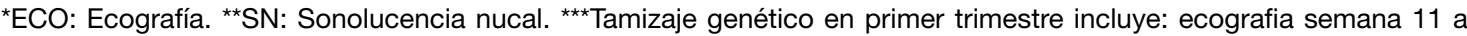
14 de gestación, cuantificación de la fracción libre de la Beta HCG, proteína plasmática asociada al embarazo. NA: No aplicable.

Dado que ese protocolo era poco eficiente se buscaron otros parámetros que mejoraran las probabilidades de diagnóstico versus los riesgos de los procedimientos invasivos como las pérdidas fetales. Así, a finales de los 80's y comienzos de los 90's, a la edad se le sumaron marcadores en suero materno durante el segundo trimestre que mejoraron considerablemente el diagnóstico prenatal de aneuploidías, pasando del marcador doble (alfafetoproteína y fracción beta de la hormona gonadotropina coriónica), al triple (el doble más estradiol) y al cuádruple (el triple más inhibina $\mathrm{A}$ ), todos estos marcadores como pruebas que mejoraban la detección de SD. En esas mismas décadas se desarrolla y masifica la ecografía obstétrica y de la mano se perfecciona la biopsia de vellosidad corial; esta última que si bien aumenta el riesgo de pérdida de los embarazos secundario al procedimiento, disminuye la edad gestacional de realizar el diagnóstico. Al ser los marcadores bioquímicos mencionados de segundo trimestre, se continua la búsqueda de pruebas que sugieran el diagnóstico de anomalías cromosómicas en el primer trimestre y se llega a los marcadores combinados (ecográficos y bioquímicos) $(9,10)$.

A finales de los 90 's y comienzos del siglo XXI el "test combinado", durante el primer trimestre, pasó a ser la mejor opción. Este consiste en un análisis de la sumatoria de resultados del grosor de la sonolucencia nucal, presencia o ausencia del hueso nasal más la medición de las concentraciones en suero materno de proteínas placentarias como fracción libre de la porción beta de la hormona gonadotropina coriónica humana y la proteína $A$ asociada a embarazo (PAPP-A), llegando con estos a tasas de detección de más del $90 \%(9,11)$. En el 2011 , se introdujo el uso del análisis de ADN fetal libre en sangre materna, el cual tiene una tasa de detección de SD de $99 \%$, con una especificidad también del $99 \%$; en la práctica, el uso de esta prueba podría resultar en una reducción significativa en los procedimientos invasivos para lograr diagnóstico prenatal de $\operatorname{SD}(8,9,12)$.

Sin embargo, en este estudio, en el que se diagnosticaron in útero 44 casos de SD, las indicaciones de los procedimientos invasivos para lograr el diagnóstico fueron en un $45,4 \%$ malformaciones anatómicas fetales diagnosticadas por ecografía, hallazgo que es similar a lo encontrado por Bornstein y cols en 2010 (13), quien compara las indicaciones de procedimientos invasivos con los cuales se hace diagnóstico de SD por trisomía libre vs. SD por otras presentaciones citogenéticas, entre los años 2000 y 2006; se reportó que de 1011 casos de trisomía 21 libre, la indicación del procedimiento invasivo fue 
anormalidades en la ecografía en el $49,7 \%$ de los casos. Frecuencia similar encontrada en los resultados de los dos estudios, uno en un país desarrollado y otro en un país en vía de desarrollo, los que muestran que la primera ayuda diagnóstica para sospechar el SD in útero es la ecografía de segundo trimestre. También, estos resultados contrastan con la evolución del diagnóstico prenatal de SD relatado en los primeros párrafos, en donde se muestra que este diagnóstico se puede lograr con indicaciones encontradas en el primer trimestre con tasas superiores al $90 \%(8-12)$.

No obstante, Bornstein y cols (13), reportan que el $6,8 \%$ de las indicaciones de los procedimientos invasivos fueron por tamizaje genético positivo en el primer trimestre y en nuestro estudio fue del $6,8 \%$, datos idénticos. Sin embargo, aquí reportamos que por la observación de marcadores ecográficos del primer trimestre (sonolucencia nucal alterada y sonolucencia nucal alterada más otro marcador ecográfico), se indicaron el $20,5 \%$ de los procedimientos invasivos que diagnosticaron SD, mostrando que las indicaciones del procedimiento invasivo se consiguieron en el $27,3 \%$ de los casos en el primer trimestre, permitiendo realizarse el procedimiento y a las pacientes tomar decisiones a más temprana edad gestacional sobre la continuidad del embarazo.

Las diferencias en los resultados entre los dos estudios radican en que Bornstein y cols (13) describen que el $37,6 \%$ de las indicaciones de los procedimientos invasivos en esos embarazos fueron por un tamizaje positivo en el segundo trimestre y en nuestro estudio ningún caso tuvo esa indicación. En contraste, en este estudio reportamos que la edad y ansiedad materna suman un $25,0 \%$ de las indicaciones en fetos con SD; mientras que Bornstein y cols no reportaron ningún feto bajo esas indicaciones. Sugiriendo estos hallazgos que en nuestra población la edad materna y la ansiedad juegan un papel importante, siendo un cuarto de las indicaciones de los procedimientos invasivos, mostrando un pobre desarrollo de las pruebas de tamizaje en diagnóstico prenatal.

A su vez, la sospecha diagnóstica se hizo en el segundo trimestre casi en la mitad de los casos. Mostrando los resultados que la primera ayuda diagnóstica para sospechar el SD es la ecografía de segundo trimestre. Es probable que estos hallazgos sean similares en otros países en Latinoamérica.

Por otro lado el análisis sobre la edad materna y el diagnóstico in útero de SD, no mostró diferencias respecto a la indicación para procedimiento invasivo para el cariotipo entre los grupos de edad materna de 34 años o menos y de 35 a 39 años. Cuando se incluye en las comparaciones el grupo de edad de 40 años y más, se encuentra que la indicación de la edad materna presenta una diferencia significativa para este subgrupo, lo cual se debe a esta indicación especial para las gestantes en esa edad. Además, se encontró un mayor valor predictivo positivo de los procedimientos invasivos para SD en el grupo de edad materna de 35 a 39 años $(22,1 \%)$ en comparación con el grupo de gestantes de 40 años y mayores (4,9\%), contrario a lo reportado en la literatura (14-16). Este hallazgo podría ser explicado porque en el grupo de 35 a 39 años, la indicación del procedimiento invasivo no fue la edad materna en ninguna de las gestantes, y las indicaciones para un diagnóstico invasivo fueron en su mayoría guiadas a partir de tamizajes positivos durante el control prenatal (80,9\%; ver Tabla I), lo cual incrementa la probabilidad de un resultado positivo en el grupo que se ha filtrado mediante el tamizaje. En contraste, en el grupo de 40 años o mayores, la edad materna fue la única indicación en la mayoría $(66,7 \%)$ de los fetos con SD; cuyo valor predictivo positivo para SD es bajo (4,3\%; ver Tabla II).

La ecografía obstétrica ha sido protocolizada en Colombia, y otros países de Latinoamérica, dentro del esquema básico de atención en el control prenatal. Por ejemplo en Colombia en la norma de atención a la embarazada emitida por el Ministerio de Salud en el año 2000 sugerían una única ecografía, entre las 20 y 24 semanas de gestación (17); mientras que en la Guía Integral de Atención en la embarazada del 2013 establece como obligatorio dos ecografías, una entre las 10 semanas más 6 días y las 13 semanas más 6 días, y otra entre las 18 semanas y 23 semanas más 6 días (18). Lográndose la masificación de esta herramienta diagnóstica que además de detectar anomalías congénitas facilita establecer la edad gestacional (19).

Esta sería una posible explicación a que en este estudio, los hallazgos ecográficos, en su conjunto, hayan sido indicación para procedimientos invasivos que llevaron al diagnóstico de SD en el $65,9 \%$ de los casos diagnosticados.

En contraste, el tamizaje bioquímico del segundo trimestre, el test combinado y el análisis del ADN fetal son pruebas que no cubre el sistema nacional de salud y los pacientes deben pagar éstos exámenes, por tanto están restringidos al segmento de población que tiene los recursos económicos necesarios. Esta limitante contribuye a que no sea una herramienta frecuentemente utilizada para el diagnóstico prenatal del SD, a diferencia de la ecografía obstétrica, obligatoriamente gratuita en toda embarazada.

\section{CONCLUSIONES}

Los resultados aquí presentados nos sugieren que no se ha logrado masificar el uso de los marcadores bioquímicos en segundo trimestre o el test combinado en el primer trimestre de gestación en nuestra población. En cambio los hallazgos 
ecográficos en el primer y segundo trimestre del embarazo alcanzaron a ser la indicación para procedimientos invasivos que llevaron al diagnóstico de SD en 2 tercios de los casos; la edad materna continúa siendo una indicación importante de los procedimientos invasivos.

\section{REFERENCIAS}

1. Montoya J, Peña A ,SatizabalJ García F. Análisis Sistémico in silico de la expresión diferencial de genes localizados en la región crítica del síndrome de Down (dscr) en el cerebro humano. Rev Fac Med 2012;20(1):15-26.

2. Ramirez R, Isaza C, Gutierrez M. La incidencia del síndrome de Down en Cali. Rev Colombia Med 1996;27:138-42.

3. Al-Biltagi M. Echocardiography in children with Down syndrome. World J Clin Pediatr 2013;2(4):36-45

4. Online Mendelian Inheritance in Man, OMIM®. Johns Hopkins University, Baltimore, MD. Número MIM: \{190685\}: \{06/09/2015\}\{25/05/2015\}: Disponible en: http://omim.org/

5. Nazer J, Cifuentes L. Estudio epidemiológico global del síndrome de Down. Rev Chil Peditr 2011;82(2):105-12.

6. Gratacós E, Nicolaides K. Clinical perspective of cellfree DNA testing for fetal aneuploidies. Rev Fetal DiagnTher 2014;35(3):151-6.

7. Li W, Wang P, Chuang C, Chang Y, Yang M, ChihYao, Chao K, Yen M. Noninvasive prenatal testing for fetal trisomy in a mixed risk factors pregnancy population. Taiwan J Obstet Gynecol 2015;54(2):122-5.

8. Norton M, Jacobsson B, Swamy G, Laurent L, Ranzini A, Brar H, Tomlinson M, Pereira L, Spitz J, Hollemon D, Cuckle H, Musci T, Wapner R. Cell-free DNA analysis for noninvasive examination of trisomy. $\mathrm{N}$ Engl J Med 2015;372(17):1589-97.

9. Borrell A, Stergiotou I. Cell-free DNA testing: inadequate implementation of an outstanding technique. Ultrasound Obstet Gynecol 2015;45: 508-11.
10. Wald N, Watt $\mathrm{H}$, Hackshaw A. Integrated screening for Down's Syndrome based on tests performed during the first and second trimesters. $\mathrm{N}$ Engl $\mathrm{J}$ Med 1999;341:461-7

11. Norwitz E, Levy B. Noninvasive Prenatal Testing: The Future Is Now. Rev Obstet Gynecol 2013;6(2):48-62.

12. Everett T, Chitty L. Cell-free fetal DNA: the new tool in fetal medicine. Ultrasound Obstet Gynecol 2015;45(5):499-507.

13. Bornstein E, Lenchner E, Donnenfeld A, Jodicke C, Keeler SM, Kapp S, Divon MY. Complete trisomy 21 vs translocation Down syndrome: a comparison of modes of ascertainment. Am J Obstet Gynecol 2010;203(4):391.e1-5.

14. Sherman S, Freeman S, Allen E, Lamb N. Risk factors for nondisjunction of trisomy 21. Cytogenet Genome Res 2005;111(3-4):273-80.

15. Nazer J, Cifuentes L, Águila A, Ureta P, Bello MP, Correa $F$, et al. Edad materna y malformaciones congénitas: Un registro de 35 años, 1970-2005. Rev Méd Chile 2007;135:1463-69.

16. Pachajoa $H$, Caicedo $C$, Saldarriaga W, Méndez F. Prevalencia de defectos congénitos en un hospital de tercer nivel en Cali (Colombia) 2004-2008. Asociación con edad materna. Rev Colomb Obstet Ginecol 2011;62(2):155-60.

17. Ministerio de Protección Social. Norma técnica para la detección temprana de las alteraciones del embarazo. 2000. Bogotá DC. Ministerio de Salud, Dirección General de Promoción y Prevención. 17 pp.

18. Ministerio de Salud y Protección Social-Colciencias. Guías de práctica clínica para la prevención, detección temprana y tratamiento de las complicaciones del embarazo, parto o puerperio. Guía No. 11-15. Bogotá, Colombia; 2013.

19. Saldarriaga W, Ruíz F, Fandiño-Losada A, Cruz M, Isaza C. Evaluation of prenatal diagnosis of congenital anomalies diagnosable by prenatal ultrasound in patients in neonatal intensive care units of Cali, Colombia. Rev Colomb Med 45(1):32-38. 